\title{
Visualization of Four Limit Cycles in Near-Integrable Quadratic Polynomial Systems
}

\author{
Pei Yu*, Yanni Zeng \\ Department of Applied Mathematics, Western University \\ London, Ontario, Canada N6A 5B7
}

\section{Abstract}

It has been known for almost 40 years that general planar quadratic polynomial systems can have four limit cycles. Recently, four limit cycles were also found in near-integrable quadratic polynomial systems. To help more people to understand limit cycles theory, the visualization of such four numerically simulated limit cycles in quadratic systems has attracted researchers' attention. However, for near-integral systems, such visualization becomes much more difficult due to limitation on choosing parameter values. In this paper, we start from the simulation of the well-known quadratic systems constructed around the end of 1979, then reconsider the simulation of a recently published quadratic system which exhibits four big size limit cycles, and finally provide a concrete near-integral quadratic polynomial system to show four normal size limit cycles.

Keywords: Hilbert's 16th problem, quadratic near-integrable system, limit cycle, Hopf bifurcation, Poincaré bifurcation, Melnikov function

MSC: 34C07; 34C23

\section{Introduction}

The well-known Hilbert's 16th problem is remained unsolved for more than one hundred years since Hilbert [6] proposed the 23 mathematical problems. A simplified version of the problem, based on a general Liénard equation, was chosen by Smale [9] as one of the 18 challenging mathematical problems for the 21st century. Consider the following planar system:

$$
\dot{x}=P(x, y), \quad \dot{y}=Q(x, y),
$$

where the dot denotes differentiation with respect to time $t, P(x, y)$ and $Q(x, y)$ are polynomials in $x$ and $y$. The second part of Hilbert's 16th problem is to find the upper bound, called Hilbert number and denoted by $H(n)$, where $n=\max \{\operatorname{deg} P, \operatorname{deg} Q\}$, on the number

\footnotetext{
* Corresponding author, Email: pyu@uwo.ca
} 
of limit cycles that system (1) can have. If the problem is restricted to the neighborhood of isolated fixed points, then the question is reduced to studying degenerate Hopf bifurcations. In 1952, Bautin [2] proved that three small limit cycles exist around a fine focus or a center in quadratic systems. Almost 30 years later, concrete examples were independently constructed by Shi [8], and by Chen and Wang [3] to show the existence of 4 limit cycles in quadratic systems, implying that $H(2) \geq 4$. However, the question whether $H(2)=4$ is still open.

To reduce the difficulty in attacking the Hilbert's 16th problem, Arnold proposed a weak version of the problem [1], which transforms the problem of determining the maximal number of limit cycles (a geometric problem) to finding the maximal number of isolated zeros of the Abelian integral or Melnikov function (an algebraic problem):

$$
M(h)=\oint_{H(x, y)=h} Q(x, y) d x-P(x, y) d y,
$$

where $H(x, y), P$ and $Q$ are all real polynomials in $x$ and $y$ with $\operatorname{deg} H=n+1$, and $\max \{\operatorname{deg} P, \operatorname{deg} Q\} \leq n$. The weak Hilbert's 16th problem is closely related to the maximal number of limit cycles in the following near-Hamiltonian system [4]:

$$
\begin{aligned}
& \dot{x}=\frac{\partial H(x, y)}{\partial y}+\varepsilon p_{n}(x, y), \\
& \dot{y}=-\frac{\partial H(x, y)}{\partial x}+\varepsilon q_{n}(x, y),
\end{aligned}
$$

where $p_{n}(x, y)$ and $q_{n}(x, y)$ are $n$ th-degree polynomials in $x$ and $y$, and $H(x, y)$ is a polynomial in $x$ and $y$ with $\operatorname{deg} H=n+1$, and $0<\varepsilon \ll 1$ indicating the small perturbations $\varepsilon p_{n}(x, y)$ and $\varepsilon q_{n}(x, y)$ on the system. When $\varepsilon=0,(3)$ is reduced to a Hamiltonian system, and the first-order ( $\varepsilon$-order) Melnikov function becomes

$$
M(h)=\oint_{H(x, y)=h} q_{n}(x, y) d x-p_{n}(x, y) d y .
$$

In this paper, we focus on quadratic systems, i.e., on the case $n=2$ in (1), and pay particular attention to the numerical realization of four limit cycles which are visualizable. In general, a quadratic system which has at least two singularities can be written in the form of $[3,10]$

$$
\begin{aligned}
& \dot{x}=-y+l x^{2}+m x y+n y^{2}, \\
& \dot{y}=x(1+a x+b y),
\end{aligned}
$$

which, under the assumption $n \neq 0$, can be rescaled to

$$
\begin{aligned}
& \dot{x}=-y+l x^{2}+m x y+y^{2}, \\
& \dot{y}=x(1+a x+b y) .
\end{aligned}
$$

System (6) is exactly the same as the system given in [12] under the transformation $(x, y) \rightarrow$ $(y, x)$ :

$$
\begin{aligned}
& \dot{x}=y\left(1+a_{1} x+a_{2} y\right), \\
& \dot{y}=-x+x^{2}+a_{3} x y+a_{4} y^{2} .
\end{aligned}
$$


Note that system (6) has two singularities at $(0,0)$ and $(0,1)$, while system (7) has two singularities at $(0,0)$ and $(1,0)$.

Recently, Kuznetsov et al. [7] considered the following quadratic system:

$$
\begin{aligned}
& \dot{x}=y+x^{2}+x y, \\
& \dot{y}=a_{2} x^{2}+b_{2} x y+c_{2} y^{2}+\alpha_{2} x+\beta_{2} y,
\end{aligned}
$$

and proved that the system has four limit cycles if certain conditions on the parameters are satisfied. In particular, they chose a set of parameter values to show four big size limit cycles.

To consider perturbing an integrable system, we need $(0,0)$ to be a center, for which it has the classifications as follows. The origin of (7) is a center if and only if one of the following conditions is satisfied [12],

$$
\begin{aligned}
& Q_{3}^{\mathrm{R}}-\text { Reversible system : } a_{3}=a_{2}=0 ; \\
& Q_{3}^{\mathrm{H}}-\text { Hamiltonian system : } a_{3}=a_{1}+2 a_{4}=0 ; \\
& Q_{3}^{\mathrm{LV}}-\text { Lokta-Volterra system : } a_{2}=1+a_{4}=0 ; \text { and } \\
& Q_{4}-\text { Codimension-4 system : } a_{3}-5 a_{2}=a_{1}-\left(5+3 a_{4}\right)=a_{4}+2\left(1+a_{2}^{2}\right)=0 .
\end{aligned}
$$

In this paper, we will perturb the reversible system to obtain the following perturbed one [12]:

$$
\begin{aligned}
\dot{x} & =y\left(1+a_{1} x\right)+\varepsilon p_{n}(x, y) \\
& =y\left(1+a_{1} x\right)+\varepsilon a_{10} x, \\
\dot{y} & =-x+x^{2}+a_{4} y^{2}+\varepsilon q_{n}(x, y) \\
& =-x+x^{2}+a_{4} y^{2}+\varepsilon\left(b_{01} y+b_{11} x y\right),
\end{aligned}
$$

where $a_{10}, b_{01}$ and $b_{11}$ are perturbation parameters.

In this paper, we consider bifurcation of limit cycles in the quadratic systems (6), (8) and (9), and show visualizable simulated four limit cycles. Since the convergence for small limit cycles are extremely slow, particularly for system (9), we apply the Runge-Kutta (R-K) 4th-order method to simulate small limit cycles on a Desktop machine with CPU@3.20GHz, and use Matlab solver ODE23 to simulate large limit cycles. For unstable limit cycles, we use backward time (i.e., using negative time step) and so the unstable limit cycles become "stable". In next section, we consider the quadratic examples proposed by Shi [8], and by Chen and Wang [3]. In Section 3, we reconsider the example constructed by Kuznetsov et al. [7] to demonstrate four big size limit cycles. Finally, we present a concrete near-integrable quadratic system [12] to show four normal size limit cycles.

\section{Four limit cycles obtained in [3] and [8]}

In this section, we consider the two concrete quadratic systems given by Shi [8] and by Chen and Wang [3]. The Shi's example is given by the following equations [8]:

$$
\begin{aligned}
& \dot{x}=\lambda x-y-10 x^{2}+(5+\delta) x y+y^{2}, \\
& \dot{y}=x+x^{2}+(-25+8 \epsilon-9 \delta) x y,
\end{aligned}
$$


where the parameter values chosen in [8] are

$$
\lambda=-10^{-250}, \quad \epsilon=-10^{-52}, \quad \delta=-10^{-13},
$$

for proving the existence of four limit cycles. The example given by Chen and Wang in [3] is described by

$$
\begin{aligned}
& \dot{x}=-\delta_{2} x-y-3 x^{2}+\left(1-\delta_{1}\right) x y+y^{2}, \\
& \dot{y}=x+\frac{2}{9} x^{2}-3 x y,
\end{aligned}
$$

where $0<\delta_{1}, \delta_{2} \ll 1$, but not specified.

Firstly it is noted that the two systems (10) and (12) have the exact same structure of (6). Secondly it was proved that both systems have a big stable limit cycle around the unstable focus $(0,1)$, and three small limit cycles around the stable focus $(0,0)$. The schematic diagrams showing the existence of limit cycles are depicted in Figure 1.

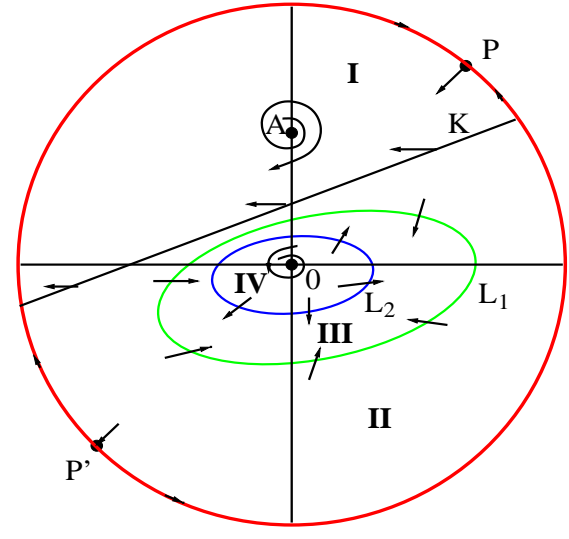

(a)

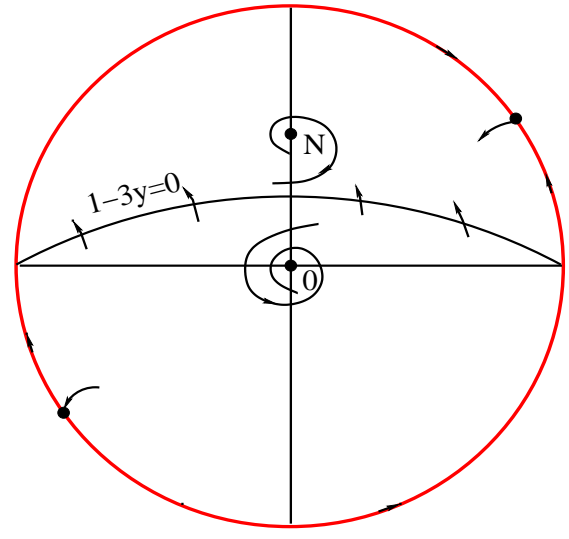

(b)

Figure 1: Schematic diagrams showing the existence of limit cycles for (a) system (10) (see Fig. 1 in [8]); and (b) system (12) (see Fig. 3 in [3]).

Note that when $\lambda=\epsilon=\delta=0$, the origin of system (10) is a 3rd-order fine focus, while the origin of system (12) is a 2nd-order fine focus when $\delta_{1}=\delta_{2}=0$. In [8] Shi explicitly constructed four trapping regions (see Figure 1(a)) and applied Poincaré-Bendixson theory to prove the existence of four limit cycles, one of them around $(0,1)$ and three of them around $(0,0)$ which were obtained by perturbing the 3rd-order fine focus using the three parameters. In [3] Chen and Wang constructed two trapping regions (see Figure 1(b)) and used Poincaré-Bendixson theory to prove the existence of the big limit cycle around $(0,1)$ and a small one around $(0,0)$. Then they showed that further perturbing the 2nd-order fine focus $(0,0)$ using the parameters $\delta_{1}$ and $\delta_{2}$ to obtain two more small limit cycles, but did not specify the values of $\delta_{1}$ and $\delta_{2}$. The stability of the four limit cycles is same for both systems: The big one around the unstable focus $(0,1)$ is stable, and the most outer small limit cycle around $(0,0)$ is unstable, the middle one is stable and the most inner one is unstable. The 
focus $(0,0)$ is stable. Figure 2 shows the simulation of the big limit cycle for systems $(10)$ and (12) when $\lambda=\epsilon=\delta=\delta_{1}=\delta_{2}=0$. Two initial points are chosen for simulating the trajectories with one outside the limit cycle (in blue color) and one inside the limit cycle (in red color), both converging to the stable limit cycle (in green color).
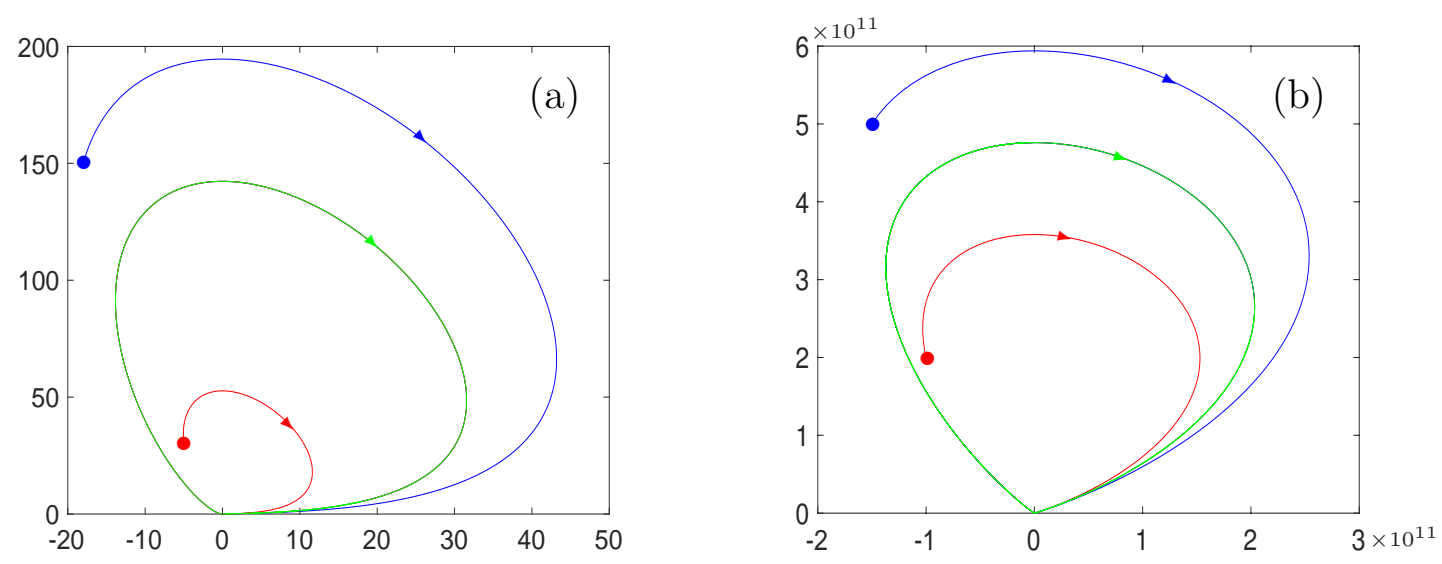

Figure 2: Simulation of big limit cycle for (a) system (10) when $\lambda=\delta=\epsilon=0$ with initial points $(-18,150)$ and $(-5,30)$; and (b) system $(12)$ when $\delta_{1}=\delta_{2}=0$ with initial points $\left(-1.5 \times 10^{11}, 5 \times 10^{11}\right)$ and $\left(-10^{11}, 2 \times 10^{11}\right)$.

To simulate the small limit cycles, the parameter values used by Shi, given in (11), to prove the existence of four limit cycles cannot be used for simulation since they are too small. When these parameters vanish, the focus values are

$$
v_{0}=v_{1}=v_{2}=0, \quad v_{3}=\frac{35625}{8} .
$$

Since $v_{3}$ is pretty large, we must choose the parameter values such that the three limit cycles are very small. To achieve this, we choose the following parameter values:

$$
\lambda=-\frac{2}{10^{8}}, \quad \epsilon=-\frac{1}{1000}, \quad \delta=-\frac{1}{10}
$$

which are much larger than that used by Shi given in (11). With these parameter values, we apply the Maple program [11] to obtain the following focus values:

$$
v_{0}=-\frac{1}{10^{8}}, \quad v_{1}=\frac{1}{10^{3}}, \quad v_{2}=-\frac{2079402109}{112500000}, \quad v_{3}=\frac{59143866813736153313}{16200000000000000} .
$$

Then, the truncated normal form up to 3rd-order terms is given by

$$
\dot{r}=v_{0}+v_{1} r^{2}+v_{2} r^{4}+v_{4} r^{6} .
$$

Solving $\dot{r}=0$ yields the approximation of the amplitudes of the three small limit cycles as follows:

$$
r_{1} \approx 0.003636, \quad r_{2} \approx 0.006431, \quad r_{3} \approx 0.070769
$$


The simulation of the three small limit cycles are shown in Figure 3, which are obtained using the R-K 4th-order method since Matlab solver ODE23 (or ODE45) takes too much time to get convergence. For a clear view, we only present the data of forming the limit cycles, but for each of the limit cycles we show two trajectories converging to the same limit cycle, one from outside the limit cycle (in blue color) and one from inside the limit cycle (in red color). It can be seen from Figure 3 that the analytical predictions agree very well with the simulations.

With the parameter values given in (13), the simulated big limit cycle is obtained using Matlab solver ODE23, as shown in Figure 4(a). Comparing this figure with Figure 2(a) shows that small perturbation parameter values can cause obvious change on the size of the limit cycle.
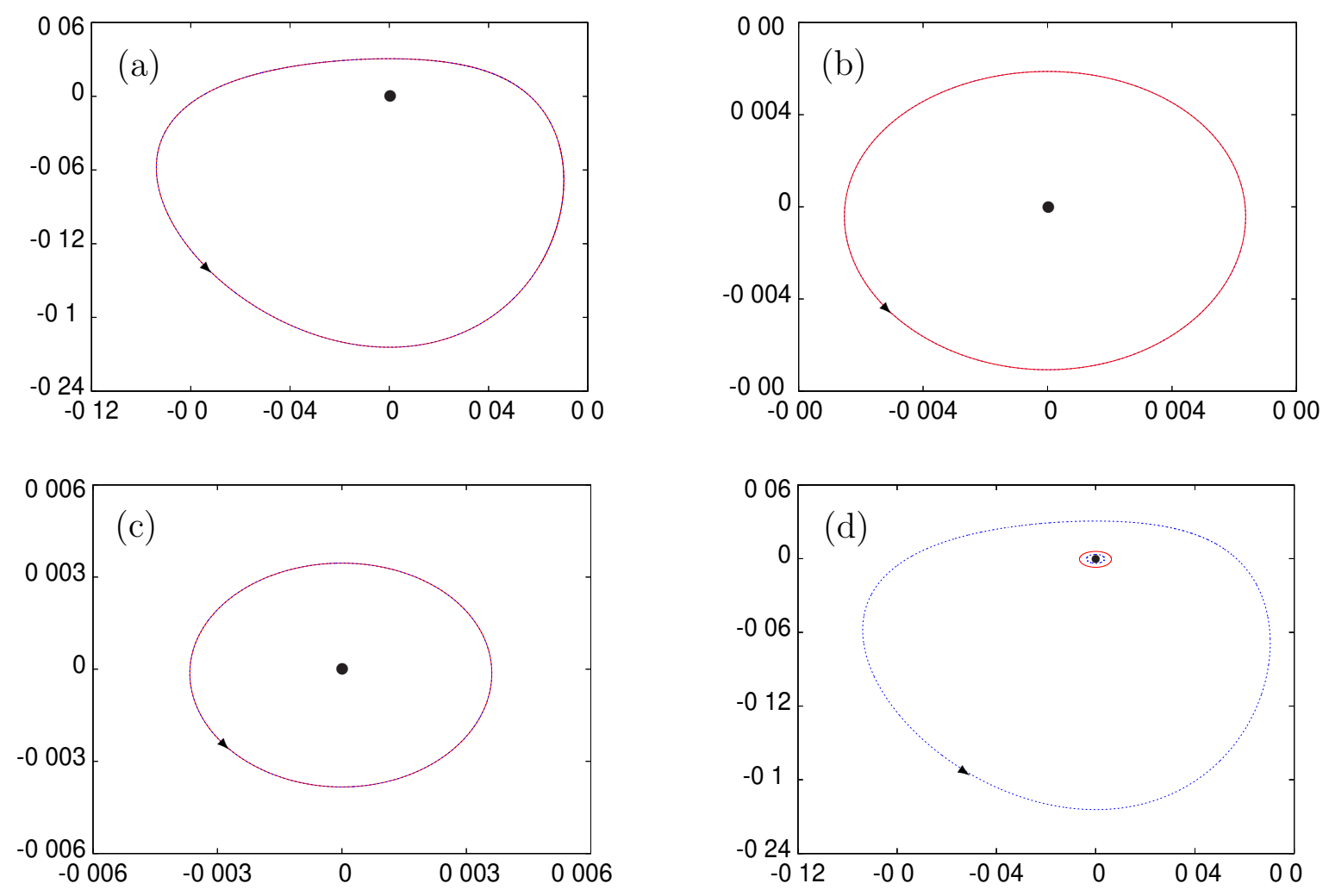

Figure 3: Simulation of 3 small limit cycles using the R-K 4th-order method for system (10) with $\lambda=-2 \times 10^{-8}, \epsilon=-0.001$ and $\delta=-0.1$ : (a) the most outer limit cycle with time step $\Delta t=-0.001$, and initial points $(0,-3)$ and $(0,0.006)$; (b) the middle limit cycle with $\Delta t=0.01$, and initial points $(0,0.006)$ and $(0,0.004) ;(\mathrm{c})$ the most inner limit cycle with $\Delta t=-0.01$, and initial points $(0,0.004),(0,0.001)$; and $(\mathrm{d})$ three small limit cycles with the stable one in red color and unstable ones in blue color.

Next, we consider the example proposed by Chen and Wang [3], given in (12). Setting $\delta_{1}=\delta_{2}=0$ yields the focus values $v_{0}=v_{1}=0, v_{2}=-\frac{77}{972} \approx-0.079218$. Thus we can 

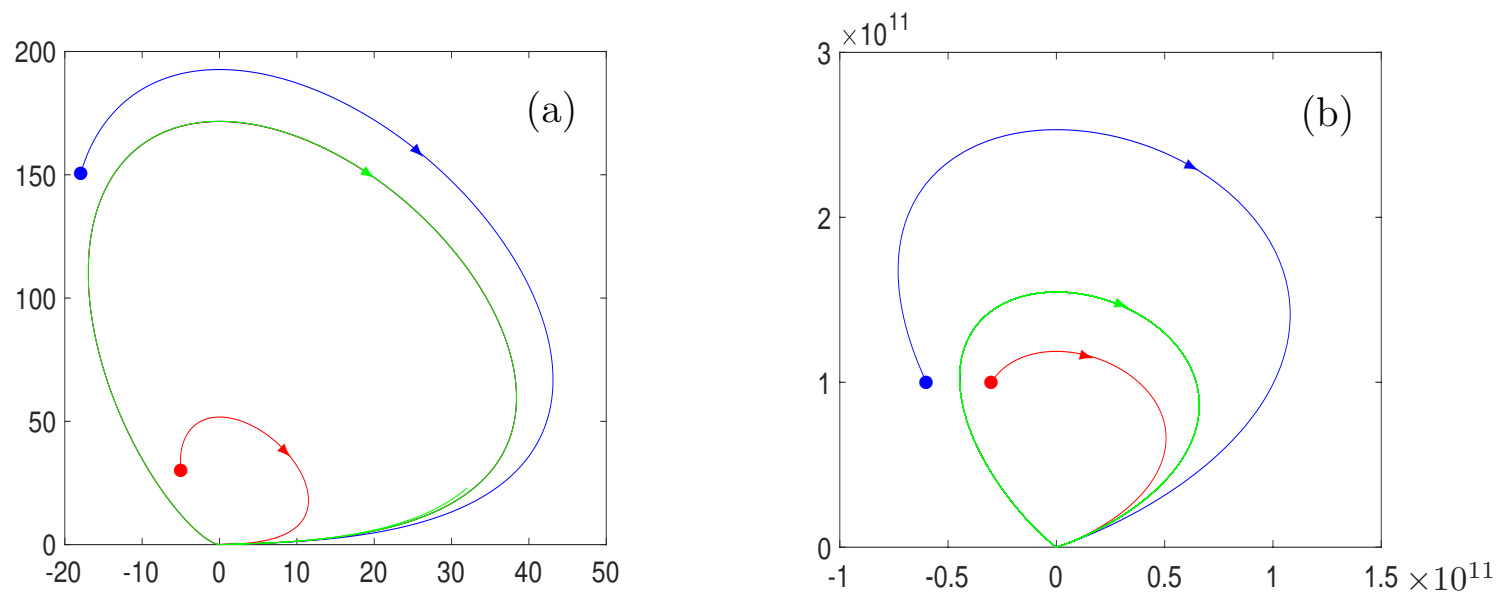

Figure 4: Simulation of the stable big limit cycle using Matlab solver ODE23: (a) for system (10) taking $\lambda=-2 \times 10^{-8}, \epsilon=-0.001, \delta=-0.1$, with initial points $(-18,150)$ and $(-5,30)$; and (b) for system (10) taking $\delta_{1}=0.01, \delta_{2}=0.00002$, with initial points $\left(-0.6 \times 10^{11}, 10^{11}\right)$ and $\left(-0.3 \times 10^{11}, 10^{11}\right)$.

choose $\delta_{1}$ and $\delta_{2}$ to obtain two small limit cycles with the outer one stable. Then by PoincaréBendixson theory, one more small unstable limit cycle can be obtained, which encloses the two small limit cycles. For this purpose, we choose

$$
\delta_{1}=0.01, \quad \delta_{2}=0.00002
$$

under which

$$
v_{0}=-10^{-5}, \quad v_{1}=0.0025, \quad v_{2}=-\frac{172948799}{2332800000} \approx-0.074138
$$

Then the truncated normal form $\dot{r}=-10^{-5}+0.0025 r^{2}-0.074138 r^{4}$ gives the approximation of the amplitudes of the two small limit cycles as $r_{1}=0.068102$ and $r_{2}=0.170538$.

With the parameter values, the simulated big limit cycle is obtained using Matlab solver ODE23, as shown in Figure 4(b). Comparing this figure with Figure 2(b) again shows that very small perturbation parameter values can have great influence on the size of the limit cycle. For this case, the limit cycle obtained with $\delta_{1}=0.01, \delta_{2}=0.00002$ is only about $1 / 3$ of that obtained with $\delta_{1}=\delta_{2}=0$.

The simulated three small limit cycles are obtained using the R-K 4th-order method, as shown in Figure 5. Again for a clear view, for each of the limit cycles we show two trajectories converging to the same limit cycle, one from outside the limit cycle (in blue color) and one from inside the limit cycle (in red color). It can be seen from this figure that the analytical predictions for the two smaller limit cycles agree very well with the simulations. 

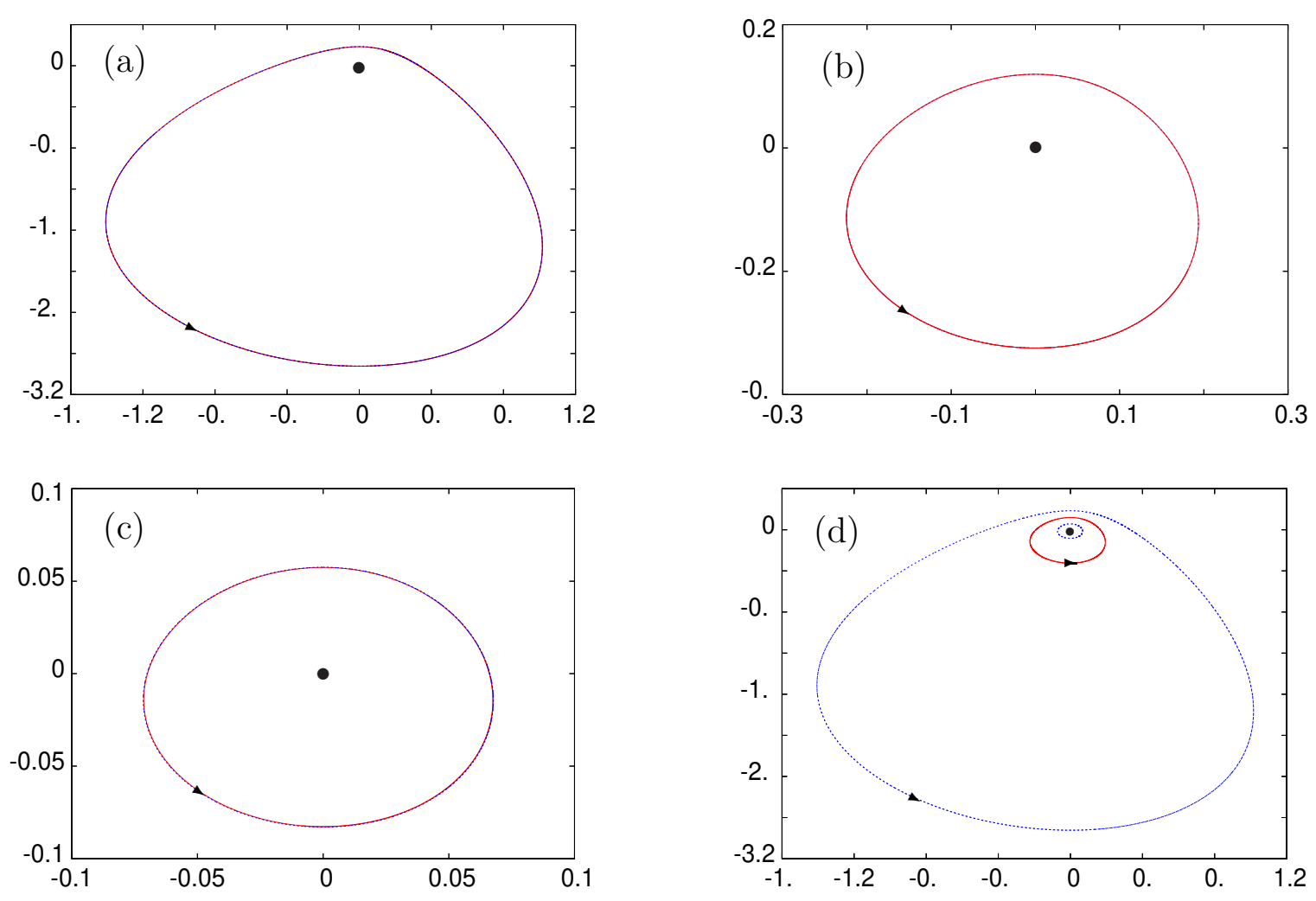

Figure 5: Simulation of three small limit cycles using the R-K 4th-order method for system (12) with $\delta_{1}=0.01$ and $\delta_{2}=0.00002$ : (a) the most outer limit cycle with time step $\Delta t=$ -0.001 , and initial points $(0,0.22)$ and $(0,0.15)$; (b) the middle limit cycle with $\Delta t=0.001$, and initial points $(0,0.15)$ and $(0,0.06)$; (c) the most inner limit cycle with $\Delta t=-0.001$, and initial points $(0,0.06),(0,0.01)$; and $(d)$ three small limit cycles with the stable one in red color and unstable ones in blue color.

\section{$3 \quad$ Four big size limit cycles obtained in [7]}

In this section, we consider system (8). In [7], Kuznetsov et al. proved that the system has four limit cycles if the following conditions hold:

$$
\begin{aligned}
& b_{2} \in(1,3), \quad c_{2} \in\left(\frac{1}{3}, 1\right), \quad 4 a_{2}\left(c_{2}-1\right)>\left(b_{2}-1\right)^{2}, \quad b_{2} c_{2}>1, \\
& \alpha_{2} \in\left(\frac{a_{2}\left(b_{2}+2\right)}{b_{2} c_{2}-1}, \frac{a_{2}\left(b_{2}+2\right)}{b_{2} c_{2}-1}+\delta\right), \quad \beta_{2} \in(0, \varepsilon), \quad 0<\varepsilon \ll \delta \ll 1 .
\end{aligned}
$$

The following parameter values:

$$
a_{2}=-10, \quad b_{2}=2.2, \quad c_{2}=0.7, \quad \alpha_{2}=-72.7778, \quad \beta=0.0015,
$$

were chosen in [7] to obtain four big size limit cycles. With the above parameter values, system (8) has two fixed points,

$$
\mathrm{E}_{0}=(0,0) \quad \text { and } \quad \mathrm{E}_{1}=(-6.2596,7.4498) .
$$


We use the parameter values in (17) and apply Matlab solver ODE23 to obtain the simulated three limit cycles around $\mathrm{E}_{0}$ as shown in Figure 6(a), and one big unstable limit cycle around $\mathrm{E}_{1}$ as shown in Figure 6(b). Note that the three limit cycles shown in Figure 6(a) are those loops between different colors. The outside red trajectory converges to the most outer limit cycle, while the blue trajectory "converges" to the middle unstable limit cycle using backward time integration, the green trajectory converges to the most inner stable limit cycle, and the inside red trajectory "converges" to the unstable focus $\mathrm{E}_{0}$ using backward time integration. In Figure 6(b), with backward time integration, two trajectories "converge" to the unstable big limit cycle (in green color), one from outside (in blue color) and one from inside (in red color).
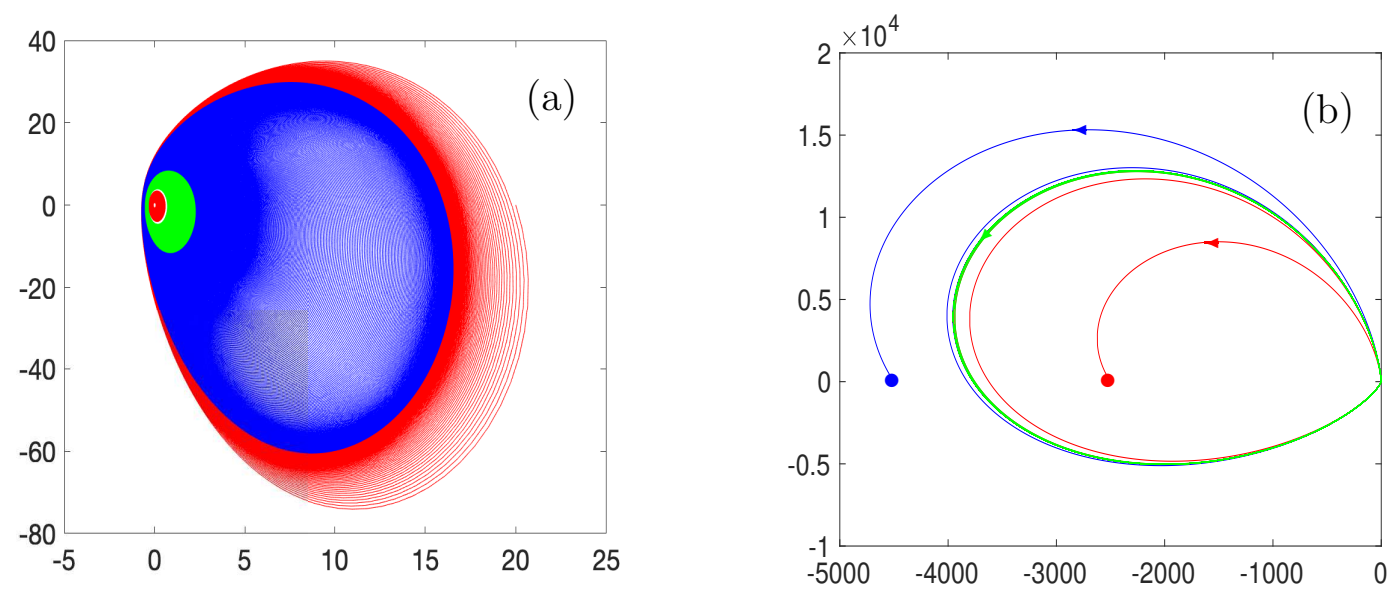

Figure 6: Simulation of limit cycles using Matlab solver ODE23 for system (8) with the parameter values given in (17): (a) three limit cycles around the unstable focus (0,0); and (b) one big unstable limit cycle (in green color) around the stable focus $(-6.2596,7.4498$ ), with two trajectories starting from the initial points $(-4500,0)$ (in blue color) and $(-2500,0)$ (in red color).

\section{Four normal size limit cycles obtained for the near- integrable system (9)}

Finally, we study the bifurcation of four limit cycles in near-integrable system (9). This system has two centers at $(0,0)$ and $(0,1)$ at $\varepsilon=0$. The Melnikov function method has been used in [12] to show that when $a_{1}<-1$, system (9) can have small limit cycles bifurcating from the two centers $(0,0)$ and $(1,0)$ with distributions: $(3,0),(0,3),(2,0),(0,2)$ and $(1,1)$. 
The Melnikov functions associated with the two centers are respectively given by

$$
\begin{aligned}
M_{0}\left(h, a_{i j}, b_{i j}\right)= & \mu_{00}\left(h-h_{00}\right)+\mu_{01}\left(h-h_{00}\right)^{2}+\mu_{02}\left(h-h_{00}\right)^{3} \\
& +\mu_{03}\left(h-h_{00}\right)^{4}+O\left(\left(h-h_{00}\right)^{5}\right), \quad \text { for } 0<h-h_{00} \ll 1, \\
M_{1}\left(h, a_{i j}, b_{i j}\right)= & \mu_{10}\left(h_{10}-h\right)+\mu_{11}\left(h_{10}-h\right)^{2}+\mu_{12}\left(h_{10}-h\right)^{3} \\
& +\mu_{13}\left(h_{10}-h\right)^{4}+O\left(\left(h_{10}-h\right)^{5}\right), \quad \text { for } 0<h_{10}-h \ll 1,
\end{aligned}
$$

where

$$
\begin{aligned}
& L_{h}: H(x, y)=h \begin{cases}\in\left(h_{00}, \infty\right), & \text { for } 1+a_{1} x>0 \\
\in\left(-\infty, h_{10}\right), & \text { for } 1+a_{1} x<0\end{cases} \\
& h_{00}=H(0,0)=\frac{1+a_{1}-a_{4}}{2 a_{4}\left(a_{1}-a_{4}\right)\left(a_{1}-2 a_{4}\right)}, \quad \text { for } 1+a_{1} x>0 \text {, } \\
& h_{10}=H(1,0)=-\frac{\left(a_{1}+1\right)\left(a_{4}+1\right)}{2 a_{4}\left(a_{1}-a_{4}\right)\left(a_{1}-2 a_{4}\right)}\left(-1-a_{1}\right)^{-\frac{2 a_{4}}{a_{1}}}, \quad \text { for } 1+a_{1} x<0 \text {, }
\end{aligned}
$$

and the coefficients $\mu_{i j}, i=0,1 ; j=0,1,2, \cdots$ can be obtained by using the Maple programs developed in [5]. It should be noted that all the coefficients $\mu_{1 k}, k=1,2, \ldots$ obtained in [5] should be multiplied by -1 , since the rotations along the loop $H(x, y)=h$ on the right side and left side of the line $x=-\frac{1}{a_{1}}$ have opposite direction. But this error does not affect the conclusion on the number of bifurcating limit cycles.

Further, the following results were proved in [12]. For the case of bifurcation of small limit cycles from the two centers $(0,0)$ and $(1,0)$ with $(3,0)$-distribution (respectively, $(0,3)$ distribution) there exists at least one large limit cycle near $L_{h}$ for some $h \in\left(-\infty, h_{10}\right)$ (respectively for some $h \in\left(h_{00}, \infty\right)$ ). For the case of limit cycles with $(2,0)$-distribution (respectively, (0,2)-distribution) there exist at least two large limit cycles, one near $L_{h_{1}}$ for some $h_{1} \in\left(-\infty, h_{10}\right)$ and one near $L_{h_{2}}$ for some $h_{2} \in\left(h_{00}, \infty\right)$. There exist corresponding values of the parameters $a_{1}$ and $a_{4}$ for the existence of four limit cycles.

Four cases were considered in [12] to yield four limit cycles, described as below.

(A) (3,0)-distribution for small limit cycles plus a $(0,1)$-distribution of large limit cycle, resulting in $(3,1)$-distribution, with the parameter values given by

$$
\begin{aligned}
& a_{1}=-\frac{30}{7}, \quad a_{4}=\frac{1}{3}\left(a_{1}-5\right)-\varepsilon_{1}, \\
& b_{11}=\frac{\left(a_{1}+2 a_{4}\right)\left(1+a_{4}-a_{1}\right)}{1+a_{4}} a_{10}-\varepsilon_{2}, \quad b_{01}=-a_{10}-\varepsilon_{3},
\end{aligned}
$$

where $\varepsilon_{k}, k=1,2,3$ are perturbation parameters.

(B) (0,3)-distribution for small limit cycles plus a (1,0)-distribution of large limit cycle, resulting in a $(1,3)$-distribution, with the parameter values given by

$$
\begin{aligned}
& a_{1}=-\frac{70}{51}, \quad a_{4}=\frac{1}{3}\left(6 a_{1}+5\right)-\varepsilon_{1}, \\
& b_{11}=\frac{\left(a_{1}+2 a_{4}\right)\left(2 a_{1}-a_{4}+1\right)}{\left(1+a_{1}\right)^{2}\left(a_{1}-a_{4}+1\right)} a_{10}-\varepsilon_{2}, \quad b_{01}=-b_{11}+\frac{2 a_{4}-1}{1+a_{1}} a_{10}-\varepsilon_{3} .
\end{aligned}
$$


(C) (2,0)-distribution for small limit cycles plus a $(1,1)$-distribution of large limit cycles, resulting in a $(3,1)$-distribution, with the parameter values given by

$$
a_{1}=-4, \quad a_{4}=-\frac{18}{5}-\varepsilon_{1}, \quad b_{11}=\frac{392}{65} a_{10}-\varepsilon_{2}, \quad b_{01}=-a_{10}-\varepsilon_{3} .
$$

(D) $(0,2)$-distribution for small limit cycles plus a $(1,1)$-distribution of large limit cycles, resulting in $(1,3)$-distribution, with the parameter values given by

$$
a_{1}=-\frac{4}{3}, \quad a_{4}=-\frac{6}{5}-\varepsilon_{1}, \quad b_{11}=\frac{1176}{65} a_{10}-\varepsilon_{2}, \quad b_{01}=-\frac{513}{65} a_{10}-\varepsilon_{3} .
$$

It should be pointed out that although the above formulas were given in [12] and the existence of large limit cycles were proved using the Melnikov function for each case, the three small limit cycles were not explicitly shown but with a proof for the existence of fine focus with a schematic plotting. In other words, the three perturbations $\varepsilon_{k}, k=1,2,3$ were not definitely defined to numerically demonstrate the three small limit cycles.

Now, we choose the formulas and parameter values given in $(\mathrm{A})$ for simulation. When $\varepsilon_{k}=0, k=1,2,3$, the focus values associated with $(0,0)$ are

$$
v_{0}=v_{1}=v_{2}=0, \quad v_{3}=\frac{347875}{7260624} \approx 0.047913 .
$$

Now we set

$$
a_{10}=0.005, \quad \varepsilon_{1}=0.1, \quad \varepsilon_{2}=3 \times 10^{-5}, \quad \varepsilon_{3}=10^{-8}
$$

which results in

$$
a_{1}=-\frac{30}{7}, \quad a_{4}=-\frac{671}{210}, \quad a_{10}=\frac{1}{200}, \quad b_{01}=-\frac{500001}{100000000}, \quad b_{11}=\frac{49182857}{968100000},
$$

and further taking $\varepsilon=\frac{1}{100}$ we have system (12) in the form of

$$
\begin{aligned}
& \dot{x}=y\left(1-\frac{30}{7} x\right)+\frac{1}{100} \times \frac{1}{200} x, \\
& \dot{y}=-x+x^{2}-\frac{671}{210} y^{2}+\frac{1}{100} \times\left(-\frac{500001}{100000000} y+\frac{49182857}{968100000} x y\right) .
\end{aligned}
$$

Then, we apply the Maple program [11] to obtain the following focus values:

$v_{0}=-\frac{1}{200000000}, \quad v_{1}=\frac{461}{56000000}, \quad v_{2}=-\frac{36481804571}{14817600000000}, \quad v_{3}=\frac{11326448548182069181}{25092716544000000000}$.

Then solving the truncated normal form (14) with the above focus values we obtain the approximated amplitudes of the three small limit cycles around $(0,0)$ :

$$
r_{1} \approx 0.026385, \quad r_{2} \approx 0.079134, \quad r_{3} \approx 0.140966
$$

However, unlike the three quadratic systems considered in the previous two sections, the simulation for this near-integrable system is extremely difficult since the convergence speed is too slow. We apply the R-K 4th-order method to simulate the system and obtain the results shown in Figures 7 and 8. Again, for each of the limit cycles we take two initial points, one from outside and one from inside the limit cycle. Also, we use the backward time in integration scheme for unstable limit cycles. The time step, the number of iterations and the CPU time are given in Table 1. 


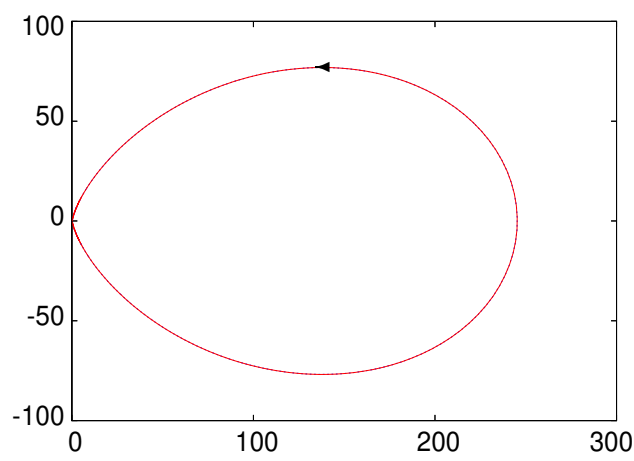

Figure 7: Simulated two trajectories for system (20), converging to the big stable limit cycle around the unstable focus $(1,0)$, with the initial points $(300,0)$ (in red color) and $(10,0)$ (in blue color).
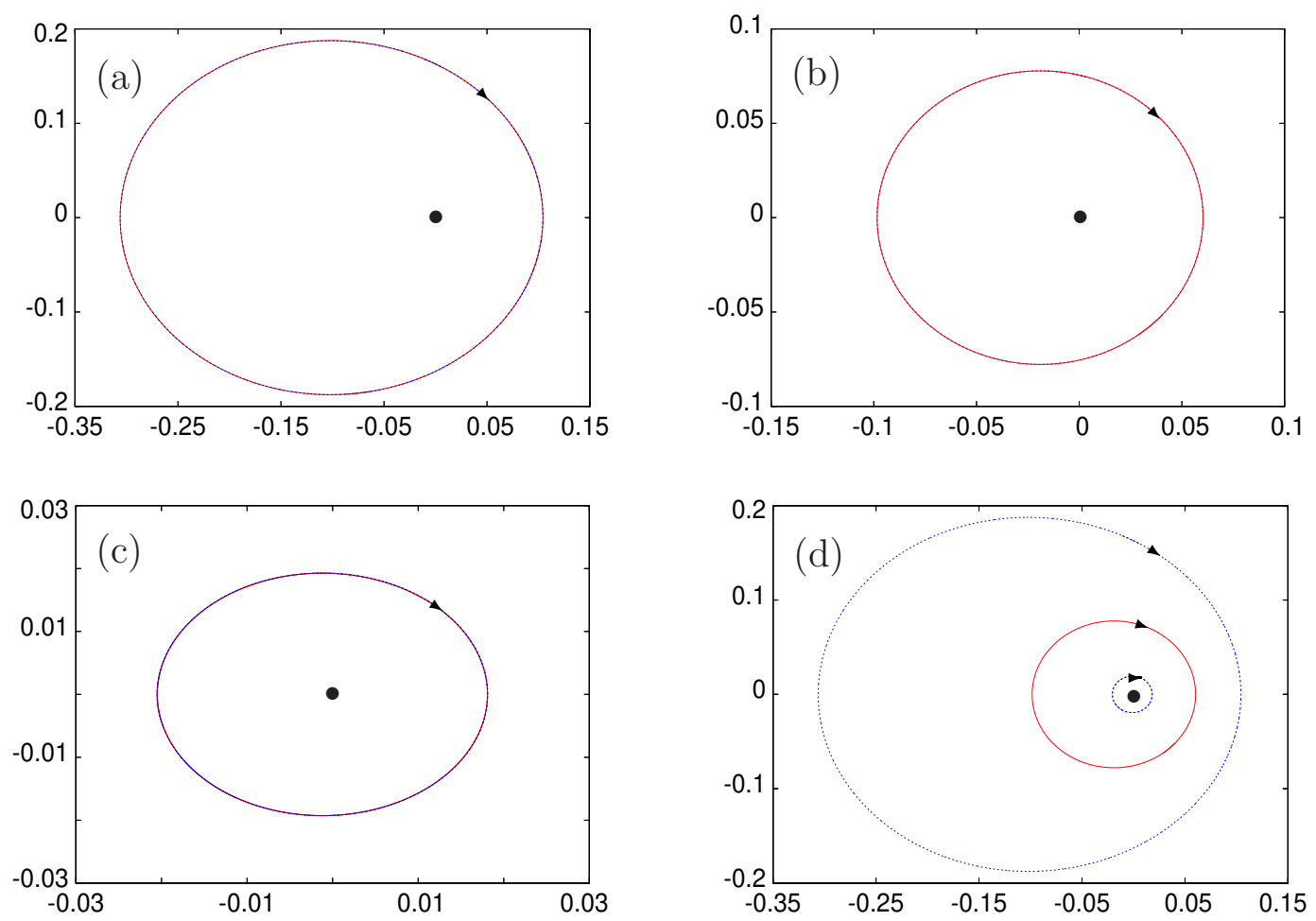

Figure 8: Simulation of the three small limit cycles for system (20): (a) two trajectories "converging" (backward time) to the most outer unstable limit cycle, from the initial points $(-0.35,0)$ (in red color) and $(-0.25,0)$ (in blue color); (b) two trajectories converging to the middle stable limit cycle, from the initial points $(-0.25,0)$ (in red color) and $(-0.05,0)$ (in blue color); (c) two trajectories "converging" (backward time) to the most inner unstable limit cycle, from the initial points $(-0.05,0)$ (in red color) and $(-0.002,0)$ (in blue color); and $(\mathrm{d})$ three limit cycles with stable one in red color and unstable ones in blue color. 
Table 1. Simulation data for system (20).

\begin{tabular}{|c|c|c|c|c|}
\hline $\begin{array}{l}\text { Trajectories } \\
\text { moving towards }\end{array}$ & $\begin{array}{l}\text { Initial } \\
\text { point }\end{array}$ & $\begin{array}{l}\text { Time } \\
\text { step }\end{array}$ & $\begin{array}{l}\text { Number of } \\
\text { interation }\end{array}$ & $\begin{array}{l}\mathrm{CPU} \\
\text { time }\end{array}$ \\
\hline$\infty$ & \multirow{2}{*}{$(300,0)$} & -0.0001 & $10^{8}$ & $2 \mathrm{sec}$ \\
\hline $\begin{array}{c}\text { The large LC } \\
\text { (Stable) }\end{array}$ & & 0.0001 & $10^{12}$ & $12 \mathrm{hr}$ \\
\hline $\begin{array}{c}(1,0) \\
\text { (Unstable) } \\
\end{array}$ & $(10,0)$ & -0.0001 & $10^{11}$ & $1.2 \mathrm{hr}$ \\
\hline$\infty$ & \multirow{2}{*}{$(-0.35,0)$} & 0.0001 & $5 \times 10^{11}$ & $6 \mathrm{hr}$ \\
\hline $\begin{array}{c}\text { The most outer LC } \\
\text { of the } 3 \text { small LCs } \\
\text { (Unstable) }\end{array}$ & & -0.001 & $10^{12}$ & $12 \mathrm{hr}$ \\
\hline $\begin{array}{l}\text { The middle LC } \\
\text { of the } 3 \text { small LCs }\end{array}$ & $(-0.25,0)$ & 0.01 & $2 \times 10^{11}$ & $25 \mathrm{hr}$ \\
\hline \multirow{2}{*}{$\begin{array}{c}\text { The most inner LC } \\
\text { of the } 3 \text { small LCs } \\
\text { (Unstable) }\end{array}$} & $(-0.05,0)$ & \multirow[t]{2}{*}{-0.02} & $8 \times 10^{12}$ & $100 \mathrm{hr}$ \\
\hline & \multirow[b]{2}{*}{$(-0.002,0)$} & & $10^{13}$ & $125 \mathrm{hr}$ \\
\hline $\begin{array}{c}(0,0) \\
(\text { Stable })\end{array}$ & & 0.02 & $7 \times 10^{12}$ & $87.5 \mathrm{hr}$ \\
\hline
\end{tabular}

\section{Conclusion}

In this paper, we have considered bifurcation of limit cycles in planar quadratic systems and numerically simulate four limit cycles which are visualizable. After we study the two well-known classical examples, and a recently published system, we focus on near-integrable systems, and construct a concrete example to show visualizable four limit cycles.

\section{Acknowledgments}

This work was supported by the Natural Sciences and Engineering Research Council of Canada (No. R2686A02). Useful discussions with Drs. Mathieu Desroches Jean-Pierre Franoise and Junmin Yang are greatly appreciated. 


\section{References}

[1] V. I. Arnold, Loss of stability of self-oscillations close to resonance and versal deformations of equivariant vector fields. Funct. Anal. Appl. 11, 85-92, 1977.

[2] N. N. Bautin, On the number of limit cycles which appear with the variation of coefficients from an equilibrium position of focus or center type. Mat. Sbornik (N.S.) 30(72), 181-196, 1952.

[3] L. S. Chen and M. S. Wang, The relative position, and the number, of limit cycles of a quadratic differential system. Acta. Math. Sinica 22, 751-758, 1979.

[4] M. Han, Bifurcation of limit cycles of planar systems. Handbook of Differential Equations, Ordinary Differential Equations, Vol. 3 (Eds. A. Canada, P. Drabek and A. Fonda), Elsevier, 2006.

[5] M. Han, J. Yang and P. Yu, Hopf Bifurcations for near-Hamiltonian Systems, Int. J. Bifurcation and Chaos 19(12), 4117-4130, 2009.

[6] D. Hilbert, Mathematical problems. (M. Newton, Transl.) Bull. Amer. Math. 8, 437479, 1902.

[7] N. V. Kuznetsov, O. A. Kuznetsova and G. A. Leronov, Visualization of four normla size limit cycles in two-dimensional polynomial quadratic system. Differ. Equ. Dyn. Syst. 21(1-2), 29-34, 2013.

[8] S. Shi, A concrete example of the existence of four limit cycles for plane quadratic systems. Sci. Sinica 23, 153-158, 1980.

[9] S. Smale, Mathematical problems for the next century. The Math. Intell. 20, 7-15, 1998.

[10] Y. Q. Ye, Some problems in the qualitative theory of ordinary differential equations. J. Diff. Eqns. 46, 153-164, 1982.

[11] P. Yu, Computation of normal forms via a perturbation technique. J. Sound Vib. 211(1), $19-38,1998$.

[12] P. Yu and M. Han, Four limit cycles from perturbing quadratic integrable systems by quadratic polynomials. Int. J. Bifurcation and Chaos 22(10), 1250254 (28 pages), 2012. 